\title{
THESEUS1 is involved in tunicamycin-induced root growth inhibition, ectopic lignin deposition, and cell wall damage- induced unfolded protein response
}

\author{
Masato Nakamura', Mamoru Nozaki ${ }^{1, a}$, Yuji Iwata², Nozomu Koizumi², \\ Yasushi Sato ${ }^{1, *}$ \\ ${ }^{1}$ Biology and Environmental Science, Graduate School of Science and Engineering, Ehime University, Matsuyama, Ehime \\ 790-8577, Japan; ${ }^{2}$ Graduate School of Life and Environmental Sciences, Osaka Prefecture University, Sakai, Osaka 599-8531, \\ Japan \\ *E-mail:ysato@sci.ehime-u.ac.jpＴel: +81-89-927-9633Fax:+81-89-927-9630
}

Received October 12, 2021; accepted December 24, 2021 (Edited by K. Suzuki)

\begin{abstract}
Endoplasmic reticulum (ER) stress activates unfolded protein responses (UPRs), such as promoting protein folding under the control of specific gene expression. Our previous study showed that ER stress induced by ER stress inducers such as tunicamycin (Tm), an inhibitor of $N$-linked glycan synthesis, causes ectopic lignin deposition in Arabidopsis roots, but the relationship between UPR and ectopic lignin deposition remains unclear. The receptor-like kinase THESEUS1 (THE1) has been shown to sense cell wall damage (CWD) induced in Arabidopsis by cellulose synthase inhibitors such as isoxaben (ISO) and to activate ectopic lignin deposition. In this study, we assessed the involvement of THE1 in ectopic lignin deposition caused by the ER stress inducer Tm. The loss-of-function mutation of THE1, the1-3, suppressed Tm-induced root growth inhibition and ectopic lignin deposition, revealing that THE1 is involved in root growth defects and ectopic lignin deposition caused by ER stress. Similarly, ISO treatment induced ectopic lignin deposition as well as the expression of the UPR marker genes binding protein 3 (BiP3) and ER-localized DnaJ $3 b$ (ERdj3b). Conversely, in the the 1-3 mutant, ISO-induced ectopic lignin deposition and the expression of BiP3 and ERdj3b were suppressed. These results showed that THE1 is involved in not only root growth inhibition and ectopic lignin deposition caused by ER stress but also CWD-induced UPR.
\end{abstract}

Key words: Arabidopsis thaliana, cell wall damage, ectopic lignin deposition, THESEUS1, UPR.

\section{Introduction}

Protein secretion is an important pathway in cell wall architecture (Liu and Howell 2016) through which nascent proteins are loaded into the endoplasmic reticulum (ER), folded properly, and subsequently transported to the Golgi apparatus. However, adverse environmental conditions can cause accumulation of unfolded or misfolded proteins in the ER, leading to cellular damage. To prevent this, ER stress activates the cellular unfolded protein response (UPR), whereby genes involved in protein folding in the ER, including binding protein 3 (BiP3) and ER-localized DnaJ $3 b$ (ERdj3b), or in the degradation of unfolded or misfolded proteins, are induced (Strasser 2018). In plants, these UPR-related genes are regulated by two signaling pathways, namely, the inositol-requiring enzyme 1 (IRE1)-basic leucine zipper 60 (bZIP60) and bZIP17/28 pathways (Fanata et al. 2013; Howell 2013; Iwata and Koizumi 2012). Additionally, UPR is involved in plant resistance to pathogen attack and heat stress (Bao and Howell 2017; Moreno and Orellana 2011).

We previously isolated the lignescens (lig) mutant of Arabidopsis thaliana (Nozaki et al. 2012), which shows a G68S point mutation in glucosamine-6-phosphate $N$ acetyltransferase (GNA1). The mutant was found to be

Abbreviations: BiP, binding protein; bZIP, basic leucine zipper; CCR, cinnamoyl CoA reductase; CESA, cellulose synthase; Col, Columbia; CrRLK1L, Catharanthus roseus RLK 1-like; CWD, cell wall damage; CWI, cell wall integrity; DMSO, dimethyl sulfoxide; ER, endoplasmic reticulum; ERdj, ERlocalized DnaJ; GM, germination medium; GNA, glucosamine-6-phosphate $N$-acetyltransferase; IRE1, inositol requiring enzyme 1; ISO, isoxaben; lig, lignescens; PME, pectin methylesterase; qRT-PCR, quantitative reverse transcription polymerase chain reaction; RBOH, respiratory burst oxidase homolog; RLK, receptor-like kinase; ROS, reactive oxygen species; SA, salicylic acid; SE, standard error; SUB, STRUBBELIG; THE1, THESEUS1; Tm, tunicamycin; UPR, unfolded protein response; WS, Wassilewskija.

a Present address: Department of Biotechnology, Graduate School of Engineering, Toyama Prefectural University, Imizu, Toyama 939-0398, Japan This article can be found at http://www.jspcmb.jp/

Published online March 5, 2022 
temperature-sensitive because of the low thermal stability of GNA1 ${ }^{\mathrm{G} 68 \mathrm{~S}}$ and was deficient in uridine diphosphate- $N$ acetylglucosamine, which is required for the formation of $\mathrm{N}$-glycans, under restrictive temperatures. Therefore, $N$-glycosylation of proteins in the ER was inhibited in the lig mutant, which caused ER stress, root growth inhibition, and ectopic lignin deposition. The phenotype of the lig mutant resembled those caused by the ER stress inducer tunicamycin (Tm), an inhibitor of $N$-linked glycan synthesis, and by dithiothreitol, a reducing agent that disrupts disulfide bonds in the wild type. These observations suggested that ER stress may be involved in root growth inhibition and ectopic lignin deposition.

Ectopic lignin deposition is associated with cell wall damage (CWD) caused by the pharmacological or genetic inhibition of cellulose synthesis (Bischoff et al. 2009; Caño-Delgado et al. 2000, 2003). A likely candidate for mediating ectopic lignin deposition by CWD is THESEUS1 (THE1), which is a receptor-like kinase (RLK) that belongs to the Catharanthus roseus RLK 1-like (CrRLK1L) protein kinase subfamily (Hématy et al. 2007; Nissen et al. 2016). Hypocotyl growth inhibition and ectopic lignin deposition in pcr1-1, which is defective in the cellulose synthesis-related subunit CESA6, were partially attenuated by a loss-of-function mutation in THE1 without influencing cellulose synthesis. Therefore, THE1 presumably mediates the response to cellulose synthesis perturbation and acts as a CWD sensor (Hématy et al. 2007).

$N$-glycosylation and processing of $N$-glycans in the secretory pathway are thought to be related to the synthesis of cellulose, which is the main load-bearing component of plant cell walls (Strasser 2014). A point mutation in $\alpha$-1,2-mannosyltransferase, whose function is to transfer mannose residues to dolichol-linked core oligosaccharides during the synthesis of $\mathrm{N}$-glycans precursors, results in defective cellulose synthesis and xylem collapse (Zhang et al. 2009). The defects in glucosidase I or glucosidase II, which process $N$-glycans in the ER, lead to reduced cellulose content (Burn et al. 2002; Gillmor et al. 2002). KORRIGAN I, a membraneanchored endo- $\beta$-1,4-glucanase, necessary for normal cellulose biosynthesis (Nagashima et al. 2018), contains eight potential $N$-glycosylation sites in the extracellular domain, and utilization of several $N$-glycosylation sites, not the structure of the attached $N$-glycan of, is important for KORRIGAN I activity (Liebminger et al. 2013). Based on this evidence, we speculated that ER stress due to a defect in $N$-glycans causes impaired cell walls.

Therefore, in this study, we hypothesized that ER stress and subsequent cell wall perturbation lead to root growth inhibition and ectopic lignin deposition via THE1 signaling. Root growth inhibition and ectopic lignin deposition by Tm treatment were reduced by THE1 mutation, confirming our hypothesis that THE1 accounts for these phenotypes induced by ER stress. Surprisingly, the the 1-3 mutant exhibited low expression of BiP3 and ERdj3b, known UPR marker genes, under Tm treatment conditions. Moreover, we found that cellulose synthesis inhibition caused by the cellulose synthesis inhibitor isoxaben (ISO) partially induced the expression of BiP3 and ERdj3b through THE1 signaling.

Overall, our study revealed that ER stress caused by defects in $N$-glycans activated THE1, leading to root growth inhibition and ectopic lignin deposition. Furthermore, we showed that THE1 signaling promoted UPR activation.

\section{Materials and methods}

\section{Plant materials}

In this study, we used several Arabidopsis thaliana mutants and strains, as described below. The double mutant bzip60,28 was obtained by crossing bzip60 (SALK_050203C) and bzip28 (SALK_132285C) in Columbia (Col)-0 background; the rbohdf (N68522) in Col-0 background was obtained from the Nottingham Arabidopsis Stock Center; and the the1-3 mutant (FLAG_201C06) in the Wassilewskija (WS) background was obtained from the Versailles Arabidopsis Stock Center.

\section{Plant culture conditions}

Sterilized seeds were treated at $4^{\circ} \mathrm{C}$ in the dark for 3 days and sown on germination medium (GM) solidified with $1.5 \%$ agar. The GM was Murashige and Skoog medium (Murashige and Skoog 1962) supplemented with $10 \mathrm{gl}^{-1}$ sucrose and buffered to $\mathrm{pH} 5.7$ with $0.5 \mathrm{gl}^{-1}$ 2-morpholinoethanesulfonic acid. Seedlings were vertically grown under continuous light (25$37 \mu \mathrm{mol} \mathrm{m}^{-2} \mathrm{~s}^{-1}$ ) at $22^{\circ} \mathrm{C}$. In liquid culture conditions, 30 to 40 seeds were sown in $1 / 10 \times$ liquid GM $(10 \mathrm{ml})$ in a $50 \mathrm{ml}$ flask and incubated on a rotary shaker at $110 \mathrm{rpm}$ under continuous light $\left(25-37 \mu \mathrm{mol} \mathrm{m}^{-2} \mathrm{~s}^{-1}\right)$ at $22^{\circ} \mathrm{C}$.

\section{Chemical treatments}

Seedlings vertically grown on plates for 5 days were transferred to plates containing $\mathrm{Tm}$ and incubated under the same conditions as the pre-culture. In liquid culture, seedlings grown in liquid medium for 4 days were directly supplied with a $2,000 \times$ concentration stock solution of Tm, ISO in dimethyl sulfoxide (DMSO), or DMSO as a mock treatment and incubated under the same conditions as used for the pre-culture.

\section{Quantitative reverse transcription (qRT)-PCR analysis}

Seedlings grown in liquid medium for 4 days were treated with either $100 \mathrm{ng} \mathrm{ml}^{-1} \mathrm{Tm}$ for $24 \mathrm{~h}$ or $600 \mathrm{nM}$ ISO for $12 \mathrm{~h}$, after which, entire seedlings were harvested for total RNA extraction. Total RNA was isolated from the seedlings as described in Chang et al. (1993), treated with DNase I (Takara Bio, Shiga, Japan), and reverse transcribed with ProtoScript II Reverse 
Transcriptase (New England Biolabs Japan, Tokyo, Japan). Firststrand CDNA was used as a template for PCR amplification of cDNA fragments. BiP3 and ERdj3b were quantified based on qRT-PCR analysis performed using the Fast Start Universal SYBR Green Master (ROX) (Roche, Basel, Switzerland) with ABI PRISM 7000 (Applied Biosystems, MA, USA) under the following conditions: an initial denaturation at $95^{\circ} \mathrm{C}$ for $10 \mathrm{~min}$, followed by 40 cycles of denaturation at $95^{\circ} \mathrm{C}$ for $15 \mathrm{~s}$, annealing, and elongation at $60^{\circ} \mathrm{C}$ for $1 \mathrm{~min}$. For quantification of cellulose synthase 5 (CESA5), CESA8, pectin methylesterase 20 (PME20), and cinnamoyl CoA reductase 2 (CCR2), qRTPCR was performed using the Luna Universal qPCR Master Mix (NEB, MA, USA) with MyGo Pro (IT-IS Life Science Ltd., Ireland) under the following conditions: an initial denaturation at $95^{\circ} \mathrm{C}$ for $1 \mathrm{~min}$, followed by 45 cycles of denaturation at $95^{\circ} \mathrm{C}$ for $15 \mathrm{~s}$, annealing, and elongation at $60^{\circ} \mathrm{C}$ for $30 \mathrm{~s}$. The gene-specific primers used in this study were as follows: ACT2 (5' -TCAGGAAGGATCTGTACGGTAAC-3' and $5^{\prime}$-TTC AGGTGGTGCAACGAC-3'), BiP3 (5'-ACCCAGCAAAGG GACTAACC- $3^{\prime}$ and $5^{\prime}$-AATACCGAGACTCAAAGGCG-3'), ERdj3b (5'-GAGGAGGCGGCATGAATAT-3' and 5' -CGT CATCCCCCTTGACA-3'), CESA5 (5'-GAGGGCACCAAA GGCACTAA-3' and 5' -CATTCCTAGCAAGCCCACCA-3'), CESA8 (5'-GCAAATTTATCATCCCAACGCTATC- ${ }^{\prime}$ ' and 5' -ATACTGACTCCGCTCCATCG-3'), PME20 (5'-GCCCTT TTCTCCAAAGGTTG- $3^{\prime}$ and $5^{\prime}$-TGCCCTTTCCGTCATCTT TA-3'), and CCR2 (5' -CCTCAGGCCGTTACATCCTC- $3^{\prime}$ and 5' -TTCTCGTCCGAACACTTGGTG-3'). The results from three technical replicates are shown. The experiments were repeated at least twice, with similar results being obtained.

\section{Phloroglucinol-HCl staining and image analysis}

Lignin staining was performed as described by Nakano and Meshitsuka (1992), with minor modifications. Seedlings grown in liquid medium for 4 days were treated with $100 \mathrm{ng} \mathrm{ml}^{-1}$ $\mathrm{Tm}$ for $72 \mathrm{~h}$ or $600 \mathrm{nM}$ ISO for $24 \mathrm{~h}$, fixed with $70 \%$ ethanol overnight at $4^{\circ} \mathrm{C}$, and then stored at $4^{\circ} \mathrm{C}$. The fixed seedlings were washed with deionized water, and the roots and cotyledons were cut into sections. These sections were stained with $1 \%(\mathrm{w} / \mathrm{v})$ phloroglucinol in $20 \%(\mathrm{w} / \mathrm{v}) \mathrm{HCl}$. The stained samples were then observed under an optical microscope (Labophot-2, Nikon, Tokyo, Japan). Lignification was compared using Ligninj (Nakamura et al. 2020). The experiments were repeatec at least twice, with similar results being obtained.

\section{Measurement of root length}

Root length was measured from seedling pictures with NeuronJ plugin (Meijering et al. 2004) using ImageJ software (Rasband 1997-2021). The experiments were repeated at least twice, with similar results being obtained.

\section{Statistical analysis}

All treatment differences were tested for significance using $\mathrm{R}$ version 3.6.3 ( $\mathrm{R}$ Core Team 2021) using $\mathrm{R}$ commander 2.62 (Fox 2005). A Shapiro-Wilk test was used to determine whether the data groups obtained in this study were normally distributed. In cases in which either or both groups showed a non-normal distribution, the significance of any difference between the two data groups was tested using the nonparametric two-sided Wilcoxon rank-sum test. In cases in which both data groups showed a normal distribution, the $F$ test was used to determine whether the variances of the data from the two groups were equal. Significant differences between the two groups with homoscedasticity were assessed using Student's $t$-test, while Welch's $t$-test was used to assess significant differences between the two groups with heteroscedasticity.

\section{Results}

Response of the the 1-3 mutant to Tm treatment To investigate the relationship between ER stress and THE1, the effects of treatment with $\mathrm{Tm}$ on root growth were examined in the the 1-3 mutant. Root growth of the 1-3 and wild-type WS was measured 3 days after Tm treatment on agar medium (Figure 1). Root growth of the 1-3 was less sensitive to Tm at 80 and $100 \mathrm{ng} \mathrm{ml}^{-1}$ compared with that of WS. Therefore, we used Tm to induce ER stress by inhibiting protein $N$-glycosylation. Thus, it was shown that THE1 may be involved in the inhibition of root growth caused by Tm-induced ER stress.

We then examined ectopic lignin deposition in the the 1-3 mutant under Tm-induced ER stress. Three-day lignin deposition in the 1-3 and WS under treatment with $100 \mathrm{ng} \mathrm{ml}^{-1} \mathrm{Tm}$ in liquid culture was analyzed by image analysis after phloroglucinol- $\mathrm{HCl}$ staining (Figure 2A, B). Significantly higher ectopic lignin deposition was detected in the roots and cotyledons of WS than in those of the 1-3 upon treatment with Tm. In addition, the expression of two UPR-related genes, BiP3, induced through the bZIP60 pathway, and ERdj3b, induced through the bZIP17/28 pathway (Ruberti et al. 2018), was analyzed using qRT-PCR (Figure 2C). The expression of $B i P 3$ and ERdj3b upon Tm treatment was significantly

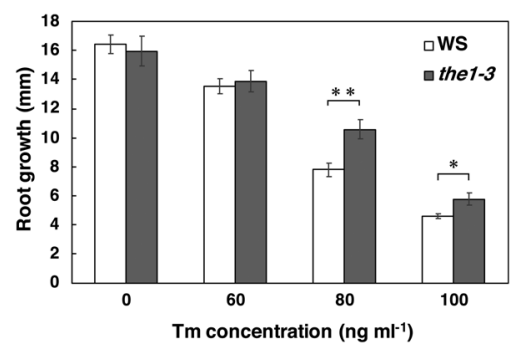

Figure 1. Effect of the ER stress inducer $\mathrm{Tm}$ on root growth in the the 1-3 mutant. Seedlings of WS and the1-3 mutant grown on vertical agar plates for 5 days were transferred to vertical agar plates containing the indicated concentrations of $\mathrm{Tm}$, and root growth was measured after 3 days. Vertical bars indicate standard error (SE) for 20 seedlings. Asterisks indicate significant differences between WS and the 1-3

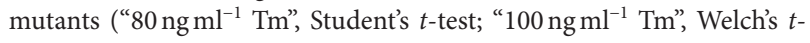
test; $\left.{ }^{*} p<0.05, * * p<0.01\right)$. 
lower in the 1-3 than in WS.

These results indicate that THE1 mediates not only the signaling pathway from ER stress caused by $N$ glycosylation inhibition to the arrest of root growth and ectopic lignin deposition in roots and cotyledons, but also the acceleration of UPR.

\section{Suppressive involvement of UPR in Tm-induced root growth inhibition and ectopic lignin deposition}

We assessed how UPR is involved in root growth
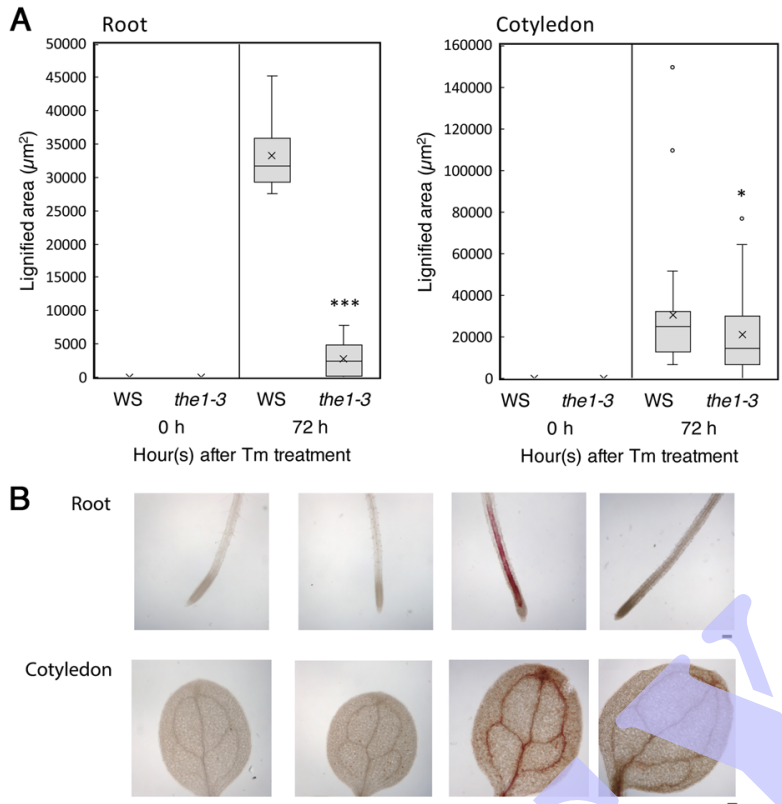

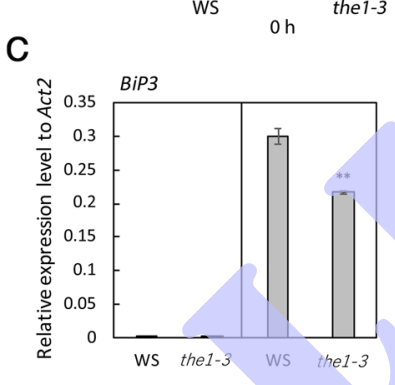

Hour(s) after Tm treat

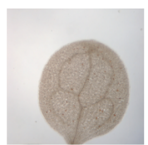

the1-3
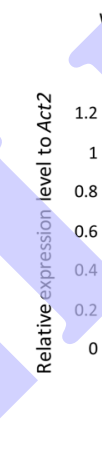

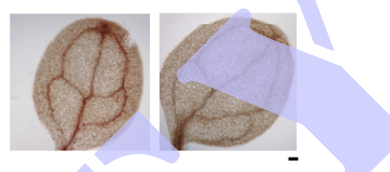

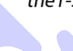

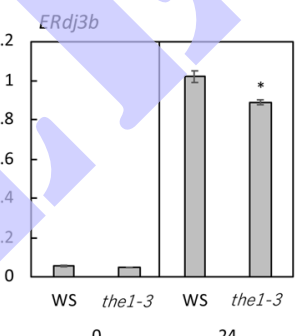

Hour(s) after Tm treatment
Figure 2. Effect of Tm treatment on ectopic lignin deposition and UPR in the the 1-3 mutant. Seedlings grown in liquid medium for 4 days were treated with $100 \mathrm{ng} \mathrm{ml}^{-1} \mathrm{Tm}$ for the indicated periods. (A) and (B) show ectopic lignin deposition in the roots and cotyledons of WS and the1-3 mutants treated with $\mathrm{Tm}$ for $72 \mathrm{~h}$. (A) Analysis of lignified area. Mean values are shown as $\times$; root: $n=5(0 \mathrm{~h}), n=8(72 \mathrm{~h})$; cotyledon: $n=10(0 \mathrm{~h}), n=25-29(72 \mathrm{~h})$. Asterisks indicate significant differences between data groups in each graph ("Root", Student's $t$-test; "Cotyledon", two-sided Wilcoxon rank-sum test; $* p<0.05$, *** $p<0.001$ ). (B) Representative pictures of roots and cotyledons stained with phloroglucinol-HCl to detect lignin. Bar $=100 \mu \mathrm{m}$. (C) qRT-PCR analysis of the expression of UPR-related genes, BiP3 and ERdj3b, in WS and the1-3 mutants treated with $\mathrm{Tm}$ for $24 \mathrm{~h}$. ACT2 was used as the standard. Asterisks indicate significant differences between WS and the 1-3 mutants ( $n=3$; bar: SE; Student's $t$-test; ${ }^{*} p<0.05$, ** $p<0.01$ ). inhibition and ectopic lignin deposition caused by the inhibition of $\mathrm{N}$-glycosylation upon treatment with Tm. Most UPR target genes in plants are regulated by the IRE1-bZIP60 and bZIP17/28 signaling pathways (Fanata et al. 2013; Howell 2013; Iwata and Koizumi 2012). Root growth of bzip60,28, a knockout mutant of bZIP60 and bZIP28, which is defective in major UPR signaling components, was significantly inhibited in the presence of 50 and $100 \mathrm{ng} \mathrm{ml}^{-1} \mathrm{Tm}$, compared to that of Col (Figure 3). Subsequently, ectopic lignin deposition and expression of UPR-related genes were analyzed under $100 \mathrm{ng} \mathrm{ml}^{-1} \mathrm{Tm}$ treatment in liquid culture (Figure 4). Ectopic lignin deposition in Tm-treated roots and cotyledons was lower in bzip60,28 than in Col (Figure $4 \mathrm{~A}, \mathrm{~B})$. In addition, the expression of BiP3 and ERdj3b induced by $\mathrm{Tm}$ treatment was not induced in the bzip60,28 mutant (Figure 4C). These results suggest that UPR alleviates Tm-induced root growth inhibition and preserves ectopic lignin deposition ability by increasing Tm tolerance.

\section{Promotion of UPR activation by CWD through THE1 signaling}

To investigate the relationship between CWD and UPR and the involvement of THE1 signaling, we used ISO, a cellulose synthesis inhibitor that activates THE1 signaling (Merz et al. 2017). We first analyzed ectopic lignin deposition induced by treatment with $600 \mathrm{nM}$ ISO for $24 \mathrm{~h}$ in WS and the 1-3 mutant (Figure 5A, B). ISO-induced ectopic lignin deposition occurred in the roots of both WS and the 1-3 and was significantly lower in the 1-3 than in WS. The results of ectopic lignin deposition in the roots of ISO-treated the 1-3 were similar to those previously reported (Engelsdorf et al. 2018; Merz et al. 2017; van der Does et al. 2017). In contrast, in the cotyledons, ectopic lignin deposition occurred in WS but rarely in the1-3. Then, the expression of BiP3 and ERdj3b in WS and the 1-3 was analyzed by qRT-PCR (Figure 5C)

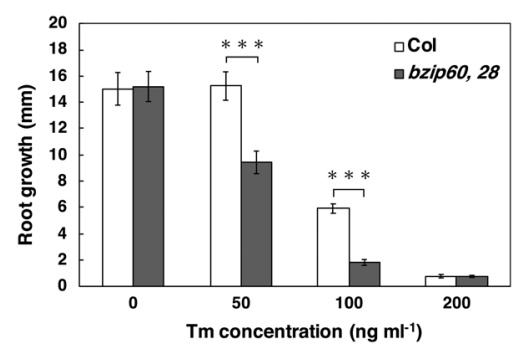

Figure 3. Effect of Tm treatment on root growth of the bzip60,28 mutant. Seedlings of $\mathrm{Col}$ and bzip60,28 mutants grown on vertical agar plates for 5 days were transferred to vertical agar plates containing the indicated concentrations of $\mathrm{Tm}$, and root growth was measured after 3 days. Vertical bars indicate SE for 20 seedlings. Asterisks indicate significant differences between $\mathrm{Col}$ and bzip60,28 mutants (" $50 \mathrm{ng} \mathrm{ml}^{-1}$

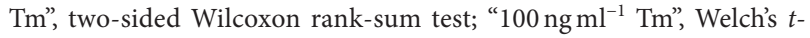
test; $* * * p<0.001)$. 
A
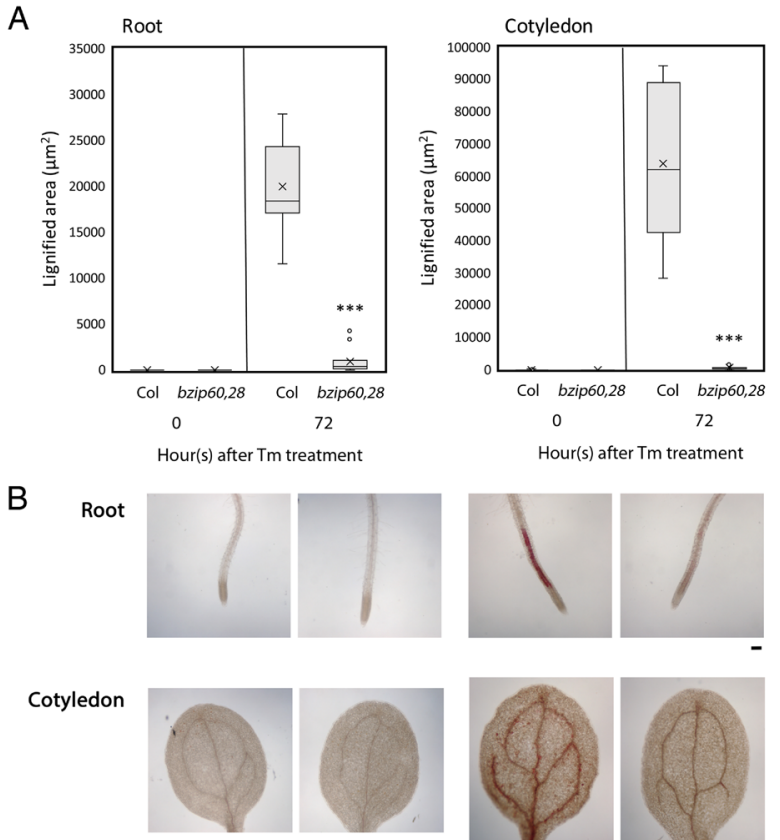

Col
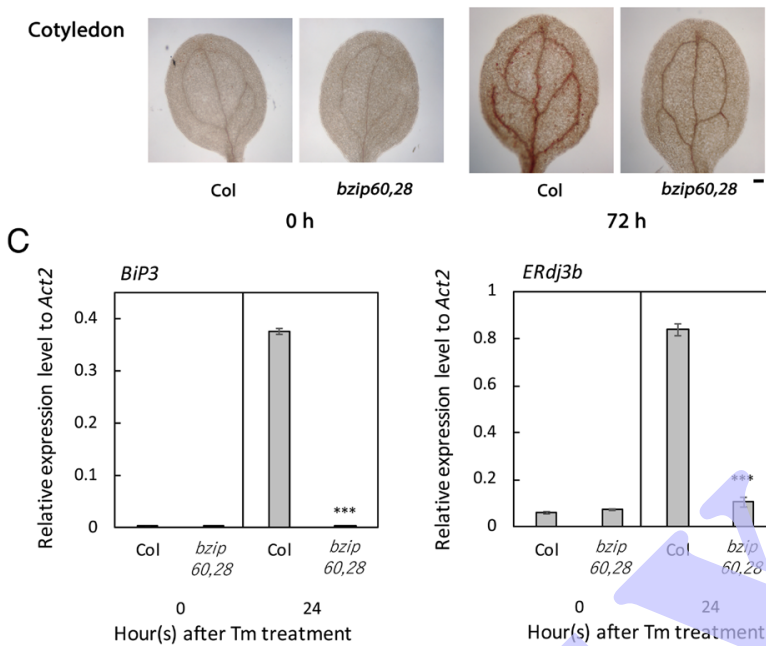

$72 \mathrm{~h}$

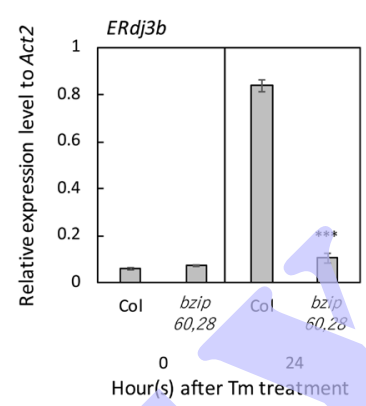

Figure 4. Effect of Tm treatment on ectopic lignin deposition and UPR in the bzip60,28 mutant. Seedlings grown in liquid medium for 4 days were treated with $100 \mathrm{ng} \mathrm{ml}^{-1} \mathrm{Tm}$ for the indicated periods. (A) and (B) show ectopic lignin deposition in the roots and cotyledons of $\mathrm{Col}$ and bzip60,28 mutants, respectively, treated with Tm for $72 \mathrm{~h}$. (A) Analysis of lignified area. Mean values are indicated by $\mathrm{X}$ : root: $n=5$ (0h), $n=9-11(72 \mathrm{~h})$; cotyledon: $n=8(0 \mathrm{~h}), n=10-12(72 \mathrm{~h})$. Asterisks indicate significant differences between data groups in each graph (two-sided Wilcoxon rank-sum test; ***p<0.001). (B) Representative pictures of roots and cotyledons stained with phloroglucinol- $\mathrm{HCl}$ to detect lignin. Bar $=100 \mu \mathrm{m}$. (C) qRT-PCR analysis of the expression of UPR-related genes, BiP3 and ERdj3b, in Col and bzip60,28 mutants treated with Tm for $24 \mathrm{~h}$. ACT2 was used as the standard. Asterisks indicate significant differences between Col and bzip60,28 mutants $(n=3$; bar: SE; "BiP3", Welch's $t$-test; “ERdj3b", Student's $t$-test; $* * * p<0.001)$.

at $12 \mathrm{~h}$ after 1 SO treatment. The expression of $B i P 3$ and ERdj3b increased at $12 \mathrm{~h}$ after ISO treatment; however, it was lower in the1-3 than in WS. These results indicate that CWD induces UPR and that THE1 signaling contributes to promoting UPR activation.

To further investigate the relationship between CWD and UPR in plants, we analyzed ectopic lignin deposition induced by $600 \mathrm{nM}$ ISO treatment for $24 \mathrm{~h}$ in $\mathrm{Col}$ and bzip60,28 mutants. ISO treatment induced
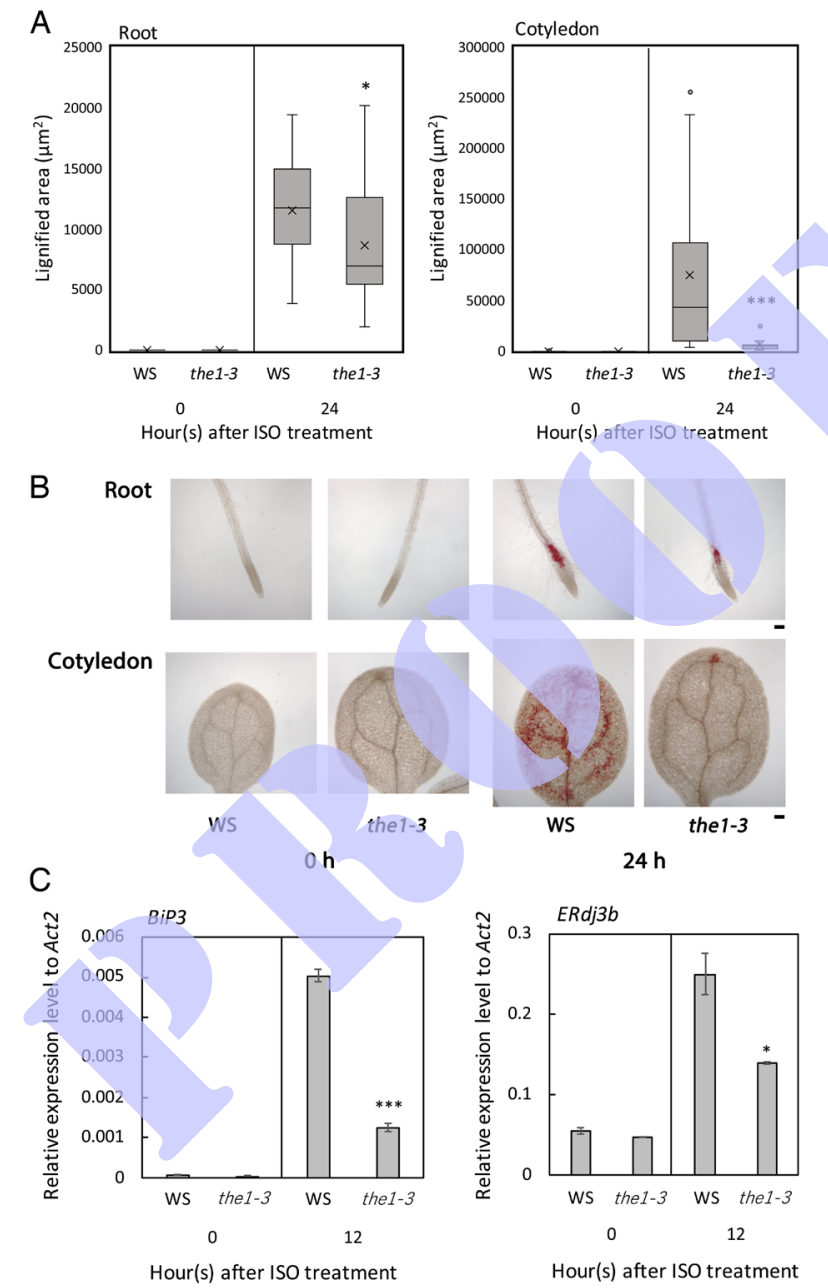

Figure 5. Effect of ISO treatment on ectopic lignin deposition and UPR in the the 1-3 mutant. Seedlings grown in liquid medium for 4 days were treated with $600 \mathrm{nM}$ ISO for the indicated periods. (A) and (B) show ectopic lignin deposition in the roots and cotyledons of WS and the1-3 mutants, respectively, treated with ISO for $24 \mathrm{~h}$. (A) Analysis of lignified area. Mean values are indicated by $\times$; root: $n=8-9(0 \mathrm{~h}), 15-$ $18(24 \mathrm{~h})$, cotyledon: $n=15-18(0 \mathrm{~h}), 19-23(24 \mathrm{~h})$. Asterisks indicate significant differences between WS and the1-3 mutants (two-sided Wilcoxon rank-sum test; $* p<0.05, * * * p<0.001$ ). (B) Representative pictures of roots and cotyledons stained with phloroglucinol-HCl to detect lignin. Bar $=100 \mu \mathrm{m}$. (C) qRT-PCR analysis of the expression of UPR-related genes, $B i P 3$ and ERdj3b, in WS and the 1-3 mutants treated with ISO for $12 \mathrm{~h}$. ACT2 was used as the standard. Asterisks indicate significant differences between WS and the 1-3 mutants $(n=3$; bar: SE; “BiP3", Student's $t$-test; “ERdj3b", Welch's $t$-test; * $p<0.05$, *** $p<0.001$ ).

ectopic lignin deposition in the roots and cotyledons of Col and bzip60,28 mutants, and no significant difference was observed between $\mathrm{Col}$ and bzip60,28 (Figure 6A, B). Then, the expression of BiP3 and ERdj3b in Col and bzip60,28 treated with ISO was analyzed by qRT-PCR (Figure 6C). In the bzip60,28 mutant, BiP3 expression was completely repressed, while $E R d j 3 b$ expression was partially downregulated. These results suggest that ISOinduced UPR did not have a significant effect on ectopic lignin deposition. 

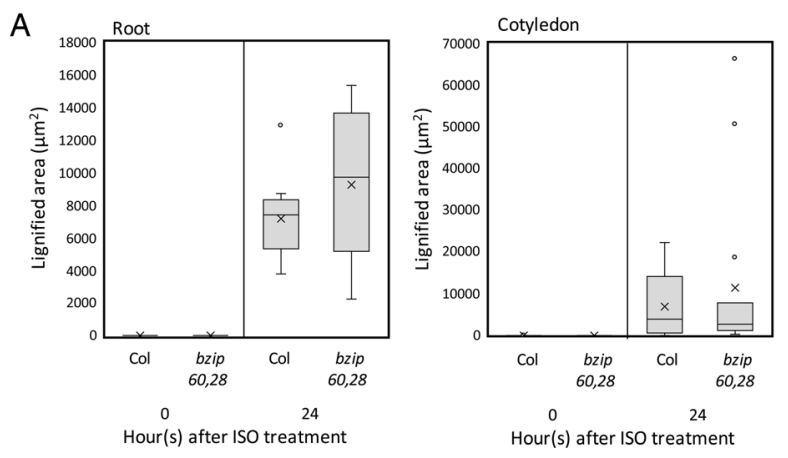

B
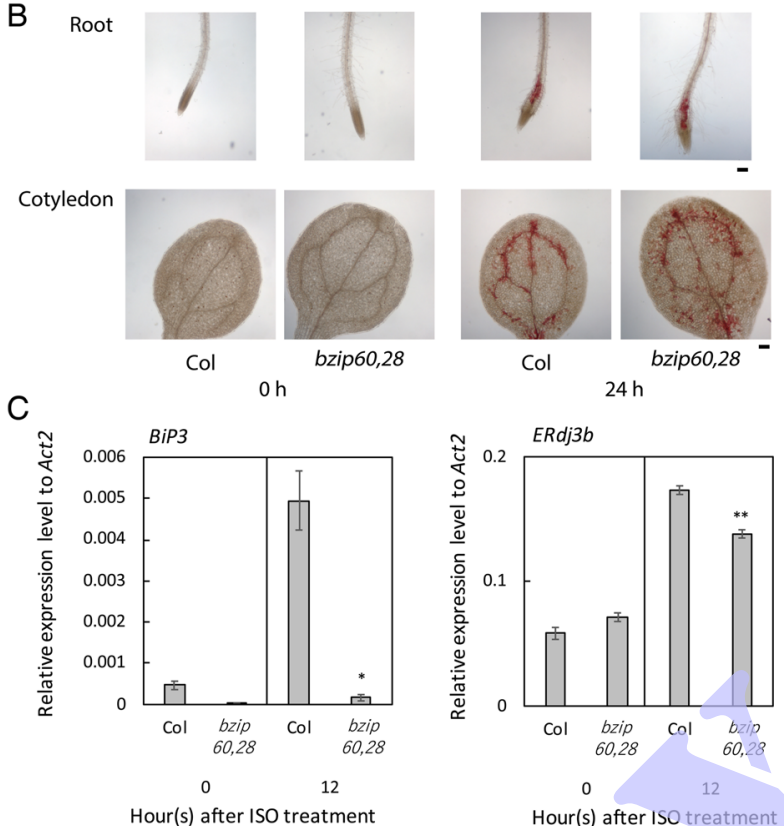

0 Hour(s) after ISO treatment

Figure 6. Effect of ISO treatment on ectopic lignin deposition and UPR in the bzip60,28 mutant. Seedlings grown in liquid medium for 4 days were treated with $600 \mathrm{nM}$ ISO for the indicated periods. (A) and (B) show ectopic lignin deposition in the roots and cotyledons of Col and bzip60,28 mutants, respectively, treated with ISO for $24 \mathrm{~h}$. (A) Analysis of lignified area. Mean values are indicated by $X$; $n=8-20$ ). (B) Representative pictures of roots and cotyledons stained with phloroglucinol-HCl to detect lignin. Bar $=100 \mu \mathrm{m}$. (C) qRT-PCR analysis of the expression of BiP3 and ERdj3b in Col and bzip60,28 mutants treated with $600 \mathrm{nM}$ ISO for $12 \mathrm{~h}$. Asterisks indicate significant differences between Col and bzip60,28 mutants ( $n=3$; bar: SE; "BiP3", Welch's $t$-test; "ERdj3b", Student's $t$-test; * $p<0.05, * * p<0.01$ ). ACT2 was used as the standard.

Comparison of cell wall synthesis-related gene expression in response to Tm and ISO treatment

ER stress due to $\mathrm{N}$-glycosylation inhibition by $\mathrm{Tm}$ is presumed to affect cell wall synthesis and induce CWD. It has been reported that CWD induced by ISO treatment alters the expression of cell wall synthesis-related genes (Bischoff et al. 2009). Therefore, we compared the expression of CESA5 as a primary cell wall synthesisrelated gene, CESA8 as a secondary cell wall synthesisrelated gene, PME20 as a pectin synthesis-related gene, and CCR2 as a stress-responsive lignin synthesisrelated gene in response to treatment with $\mathrm{Tm}$ and ISO
(Figure 7). The expression of CESA5 by ISO treatment was increased two-fold in all strains, while that by $\mathrm{Tm}$ treatment increased only in the bzip60,28 mutant. The increased expression in the bzip60,28 mutant suggests that UPR suppresses upregulation of CESA5 by Tm treatment. The expression of CESA 8 by ISO treatment was increased in all strains, while that by $\mathrm{Tm}$ treatment increased only in WS and the the 1-3 mutant. The expression of PME2O by ISO treatment was markedly increased in all strains, and that by Tm treatment also increased in WS, the the1-3 mutant, and Col. These results are consistent with those of a previous study in which PME20 expression was upregulated by CWD induced by ISO treatment (Bischoff et al. 2009). CCR2 expression was markedly increased in the $\mathrm{ISO}$ and $\mathrm{Tm}$ groups. The overall pattern of expression by ISO and $\mathrm{Tm}$ in wild-type WS and Col was similar, suggesting that CWD also occurs by Tm treatment. In addition, the expression of CCR2 was suppressed by $\mathrm{Tm}$ and ISO treatments in the the 1-3 mutant, consistent with the results presented in Figures 2 and 5, showing the suppression of lignin deposition in the the 1-3 mutant.

\section{Non-involvement of RBOH D-and F-dependent ROS and ectopic lignin deposition in UPR activation}

NADPH oxidase produces reactive oxygen species (ROS) downstream of CrRLK1L signaling (Cheung and $\mathrm{Wu}$ 2011; Galindo-Trigo et al. 2016). Arabidopsis has 10 genes encoding NADPH oxidases, known as respiratory burst oxidase homologs (RBOHs) (Torres and Dangl 2005). Among them, RBOH D and F act downstream of THE1 signaling and are seemingly involved in ectopic lignin deposition (Denness et al. 2011). Moreover, low concentrations of ROS induce ER stress-related genes (Ozgur et al. 2015).

To investigate whether $\mathrm{RBOH} \mathrm{D}$ and $\mathrm{F}$ are involved in promoting ectopic lignin deposition and UPR activation, seedlings of rbohdf and Col grown in liquid medium for 4 days were treated with $100 \mathrm{ng} \mathrm{ml}^{-1} \mathrm{Tm}$ or $600 \mathrm{nM}$ ISO, and lignin deposition and the expression of BiP3 and ERdj3b were analyzed. The rbohdf mutant did not accumulate ectopic lignin in the roots or cotyledons after Tm or ISO treatment (Figure $8 \mathrm{~A}$ ). These results support those of a previous study, which showed that ROS produced by RBOH D and $\mathrm{F}$ are required for lignin deposition in response to CWD caused by ISO (Denness et al. 2011). In contrast, the expression of BiP3 and ERdj3b induced by Tm or ISO treatment did not differ much between rbohdf and Col (Figure 8B, C). This result is consistent with that of Angelos and Brandizzi (2018) who reported that $\mathrm{Tm}$ treatment also induced BiP3 expression in the rbohdf mutant. Taken together, these results suggest that UPR activation is not caused by ROS produced by $\mathrm{RBOH} \mathrm{D}$ and $\mathrm{F}$ and the associated ectopic 

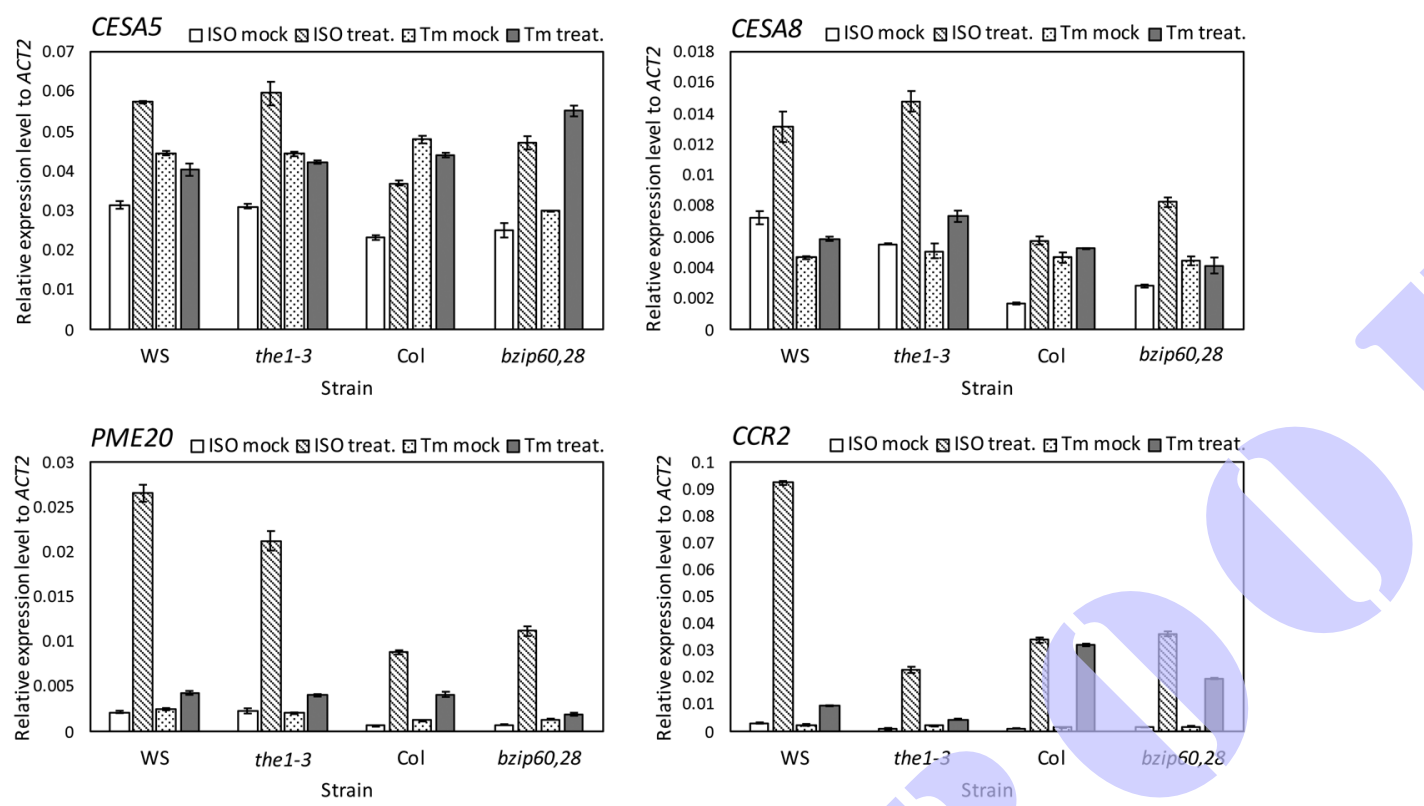

Figure 7. Effects of ISO and Tm treatments on the expression of the cell wall synthesis-related genes CESA5, CESA8, PME20, and CCR2 in WS, the1-3 mutant, Col, and bzip60,28 seedlings. Seedlings grown in liquid medium for 4 days were treated with $600 \mathrm{nM}$ ISO or DMSO (mock) for $12 \mathrm{~h}$ or $100 \mathrm{ng}^{-1} \mathrm{Tm}$ or DMSO for $24 \mathrm{~h}$. ACT2 was used as a standard ( $n=3$; bar: SE).

lignin deposition but by other factors downstream of THE1.

\section{Discussion}

\section{A model of THE1 function under ER stress}

Inhibition of $\mathrm{N}$-glycosylation by Tm treatment induces root growth inhibition, ectopic lignin deposition, and UPR (Nozaki et al. 2012). However, the factors that mediate this effect remain unclear. In this study, we found that THE1 mediated root growth inhibition and ectopic lignin deposition and promoted UPR activation caused by $\mathrm{Tm}$-induced inhibition of $\mathrm{N}$-glycosylation. THE1 is known to be a receptor kinase required for the response to inhibition of cellulose synthesis, and its activation is suggested to be induced by CWD (GalindoTrigo et al. 2016; Lindner et al. 2012; van der Does et al. 2017). Additionally, cellulose content decreases when $\mathrm{N}$-glycosylation is compromised (Strasser 2014). Based on these findings, together with the results of the present study, we propose a model to account for the function of THE1 under ER stress due to impaired $N$-glycosylation (Figure 9). ER stress due to Tm-induced impairment of $N$-glycosylation seemingly leads to CWD, which in turn inhibits root growth and promotes ectopic lignin deposition via THE1. Conversely, Tm-induced UPR alleviates ER stress by promoting protein folding in the ER, thereby mitigating the inhibitory effects of ER stress on ectopic lignification (Figure 4). Moreover, by alleviating ER stress, Tm-induced UPR also mitigates the inhibition of root growth. RBOH D and F were shown to be involved in ectopic lignin deposition but not in UPR activation by Tm or ISO. As the promotion of UPR activation by CWD also occurs in part in the1-3, it would seem that THE1 is not the sole factor involved. Thus, for example, MIK2 has been suggested to function downstream of THE1 (Engelsdorf et al. 2018; Gigli-Bisceglia et al. 2020; van der Does et al. 2017), and recently, STRUBBELIG (SUB) has been shown to be involved in cell wall integrity (CWI) independent of THE1 (Chaudhary et al. 2020), suggesting that other factors, such as SUB, may also be involved in the promotion of UPR activation by CWD.

Promotion of UPR activation via THE1 signaling in ISO-induced CWD, and involvement of UPR in ectopic lignin deposition

Analysis of wild type and the1-3 mutant suggested that ISO-induced CWD promoted UPR activation through THE1 signaling (Figure 5C). In yeast, cell wall stress activates UPR (Krysan 2009; Scrimale et al. 2009). An interrelationship between CWI and UPR has also been reported in the rice blast fungus, Magnaporthe oryzae (Yin et al. 2016). In the present study, we showed, to our knowledge for the first time in plants, that CWD activates UPR and that THE1 signaling is involved in the process. However, it should also be considered that the induction level of UPR-related genes was much lower by ISO than by Tm, a direct ER stress inducer. UPR was not as strongly induced by ISO as that by $\mathrm{Tm}$ treatment, suggesting no large difference in ectopic lignin deposition between $\mathrm{Col}$ and bzip60,28 mutants (Figure $6 \mathrm{~A}, \mathrm{~B})$. Overall, this study revealed the interrelationship of CWD, CWI, and UPR in plants. 
A

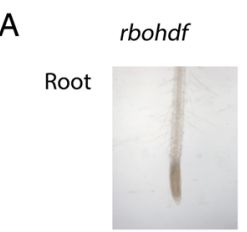

$\mathrm{Oh}$

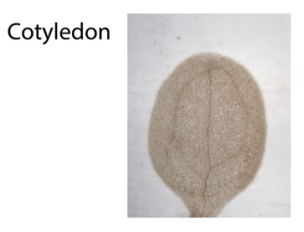

$\mathrm{Oh}$

$B$

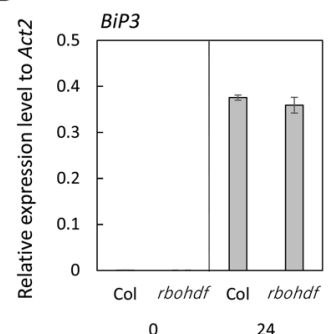

C

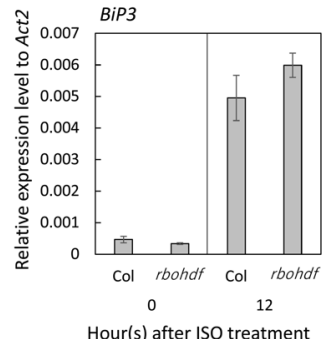

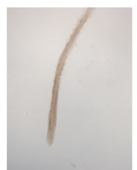

$\operatorname{Tm} 72 \mathrm{~h}$

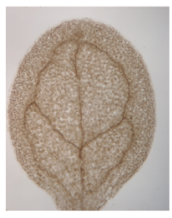

$\operatorname{Tm} 72 \mathrm{~h}$
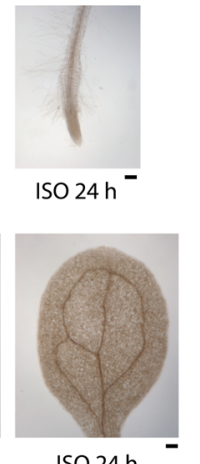

ISO $24 \mathrm{~h}$

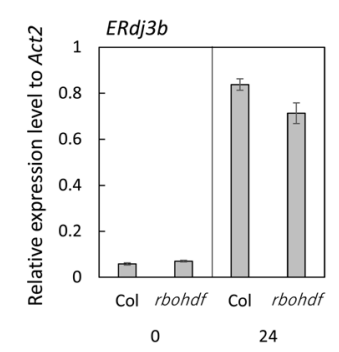

Hour(s) after Tm treatment

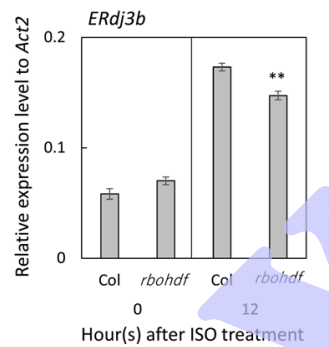

Figure 8. Effect of Tm and ISO treatments on ectopic lignir deposition and UPR in the rbohdf mutant. Seedlings grown in liquid medium for 4 days were treated with $100 \mathrm{ng} \mathrm{ml}^{-1} \mathrm{Tm}$ or $600 \mathrm{nM}$ ISO for the indicated periods. (A) Representative pictures of the roots and cotyledons of $\mathrm{Col}$ and rbohdf mutants stained with phloroglucinol$\mathrm{HCl}$ for detection of lignin. Bar $=100 \mu \mathrm{m}$. (B, C) qRT-PCR analysis of the expression of $B i P 3$ and $E R d j 3 b$ in $\mathrm{Col}$ and rbohdf mutants treated with $100 \mathrm{ng} \mathrm{ml}^{-1} \mathrm{Tm}$ for $24 \mathrm{~h}(\mathrm{~B})$ or $600 \mathrm{nM}$ ISO for $12 \mathrm{~h}(\mathrm{C})$. Asterisk indicates significant difference between $\mathrm{Col}$ and $r$ rohdf mutants $(n=3$; bar: SE; Student's $t$-test; $* * p<0.01$ ). ACT2 was used as the standard.

\section{Possible factors mediating the promotion of UPR} activation downstream of THE1 signaling

We considered ROS produced by RBOH D and F as putative factors triggering UPR downstream of THE1 signaling. Our data showed that rbohdf did not cause ectopic lignin deposition under Tm or ISO treatments because of the lack of ROS required for monolignol polymerization (Figure 8A). However, the expression of $B i P 3$ and $E R d j 3 b$ was not affected by rbohdf mutation (Figure 8B, C). Consistently, Angelos and Brandizzi (2018) reported that Tm treatment induced the expression of BiP3 in the wild type as well as in rbohdf. Altogether, these results suggest that ROS do not contribute to the promotion of UPR by Tm or

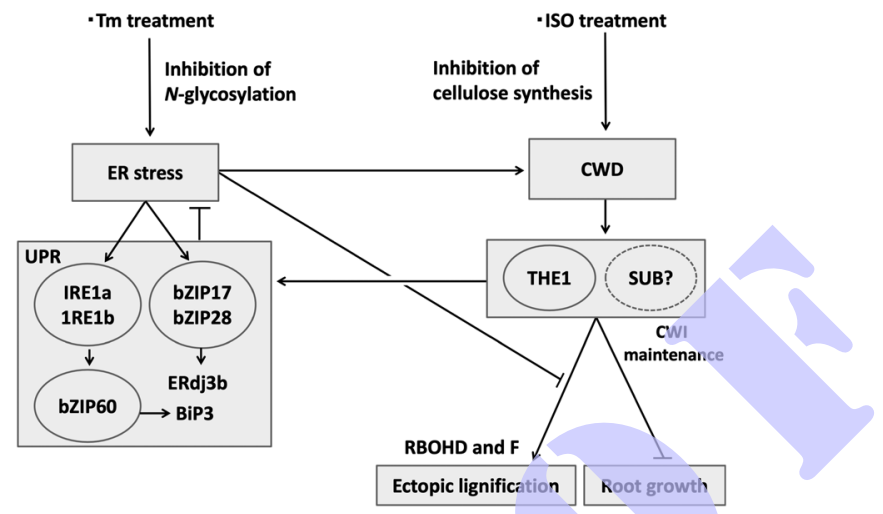

Figure 9. Proposed model showing the role of THE1 in ectopic lignin deposition in Tm- and ISO-treated seedlings. The model shows the involvement of THE1 and UPR in root growth inhibition and ectopic lignin deposition under ER stress caused by Tm-induced inhibition of $\mathrm{N}$-glycosylation. The model also shows the involvement of THE1 and UPR in CWD caused by ISO-induced inhibition of cellulose synthesis.

ISO treatment. In contrast, salicylic acid (SA), a plant hormone, induces UPR gene expression (Moreno et al. 2012; Nagashima et al. 2014). Similarly, ISO treatment increases SA accumulation in the wild type but reduces SA accumulation in THE1 loss-of-function mutants and increases SA accumulation in THE1 gain-of-function mutants (Engelsdorf et al. 2018; Gigli-Bisceglia et al. 2018; Hamann et al. 2009). These results suggest that SA may be a candidate factor that functions downstream of THE1 signaling to promote UPR activation under Tm and ISO treatments. SUB has also been shown to act as a CWD sensor (Chaudhary et al. 2020), and it is possible that SUB signaling is also involved in promoting UPR, in addition to THE1 signaling.

In this study, we showed that THE1 is involved in root growth inhibition and ectopic lignin deposition induced by ER stress through the inhibition of $\mathrm{N}$-glycosylation. Furthermore, we showed that UPR is promoted in response to CWD and that THE1 is also involved in this process. Thus, this study expands our understanding of the role of THE1 in ER stress and CWD. Several components upstream and downstream of THE1 signaling have been identified recently (Engelsdorf et al. 2018; Gigli-Bisceglia et al. 2018; Gonneau et al. 2018). Future studies will surely reveal additional details of the mechanisms underlying the interrelationship between UPR and CWI.

\section{Acknowledgements}

We thank the Nottingham Arabidopsis Stock Center and Versailles Arabidopsis Stock Center for providing the T-DNA insertion lines. We also thank Dr. Yo Sakuma of Ehime University for allowing us to use his experimental equipment and Naoto Nakatsuma of Ehime University for research assistance. This work was supported in part by JSPS KAKENHI, grant number 25440139 to Y.S. 


\section{Accession numbers}

Sequence data from this article can be found in the GenBank/ EMBL data libraries under accession numbers THESEUS1, AT5G54380; bZIP60, AT1G42990; bZIP28, AT3G10800; BiP3, AT1G09080; ERdj3b, AT3G62600; CESA5, AT5G09870; CESA8, AT4G18780; PME20, AT2G47550; CCR2, AT1G80820; RBOHD, AT5G47910; RBOHF, AT1G64060; and ACT2, AT3G18780.

\section{References}

Angelos E, Brandizzi F (2018) NADPH oxidase activity is required for ER stress survival in plants. Plant J 96: 1106-1120

Bao Y, Howell SH (2017) The unfolded protein supports plant development and defense as well as responses to abiotic stress. Front Plant Sci 8: 344

Bischoff V, Cookson SJ, Wu S, Scheible WR (2009) Thaxtomin A affects CESA-complex density, expression of cell wall genes, cell wall composition, and causes ectopic lignification in Arabidopsis thaliana seedlings. J Exp Bot 60: 955-965

Burn JE, Hurley UA, Birch RJ, Arioli T, Cork A, Williamson RE (2002) The cellulose-deficient Arabidopsis mutant $r s w 3$ is defective in a gene encoding a putative glucosidase II, an enzyme processing $\mathrm{N}$-glycans during ER quality control. Plant $\mathrm{J} 32$ : 949-960

Caño-Delgado AI, Metzlaff K, Bevan MW (2000) The eli1 mutation reveals a link between cell expansion and secondary cell wall formation in Arabidopsis thaliana. Development 127: 3395-3405

Caño-Delgado AI, Penfield S, Smith C, Catley M, Bevan M (2003) Reduced cellulose synthesis invokes lignification and defense responses in Arabidopsis thaliana. Plant J 34: 351-362

Chang S, Puryear J, Cairney J (1993) A simple and efficient method for isolating RNA from pine trees. Plant Mol Biol Report 11: 113-116

Chaudhary A, Chen X, Gao J, Leśniewska B, Hammerl R, Dawid C, Schneitz K (2020) The Arabidopsis receptor kinase STRUBBELIG regulates the response to cellulose deficiency. PLoS Genet 16: e1008433

Cheung AY, Wu HM (2011) THESEUS1, FERONIA and relatives: A family of cell wall-sensing receptor kinases? Curr Opin Plant Biol 14: 632-641

Denness L, McKenna JF, Segonzac C, Wormit A, Madhou P, Bennett M, Mansfield J, Zipfel C, Hamann T (2011) Cell wall damage-induced lignin biosynthesis is regulated by a reactive oxygen species-and jasmonic acid-dependent process in Arabidopsis. Plant Physiol 156: 1364-1374

Engelsdorf T, Gigli-Bisceglia N, Veerabagu M, Mckenna JF, Vaahtera L, Augstein F, van der Does D, Zipfel C, Hammann T (2018) The plant cell wall integrity maintenance and immune signaling systems cooperate to control stress responses in Arabidopsis thaliana. Sci Signal 11: eaao3070

Fanata WID, Lee SY, Lee KO (2013) The unfolded protein response in plant: A fundamental adaptive cellular response to internal and external stresses. J Proteomics 93: 356-368

Fox J (2005) The R Commander: A basic statistics graphical user interface to R. J Stat Softw 14: 1-42

Galindo-Trigo S, Gray JE, Smith LM (2016) Conserved roles of CrRLK1L receptor-like kinases in cell expansion and reproduction from algae to angiosperms. Front Plant Sci 7: 1269

Gigli-Bisceglia N, Engelsdorf T, Hamann T (2020) Plant cell wall integrity maintenance in model plants and crop species-relevant cell wall components and underlying guiding principles. Cell Mol
Life Sci 77: 2049-2077

Gigli-Bisceglia N, Engelsdorf T, Strnad M, Vaahtera L, Khan GA, Yamoune A, Alipanah L, Novák O, Persson S, Hejatko J, et al. (2018) Cell wall integrity modulates Arabidopsis thaliana cell cycle gene expression in a cytokinin- and nitrate reductasedependent manner. Development 145: dev166678

Gillmor CS, Poindexter P, Lorieau J, Palcic MM, Somerville C (2002) $\alpha$-glucosidase I is required for cellulose biosynthesis and morphogenesis in Arabidopsis. J Cell Biol 156: 1003-1013

Gonneau M, Desprez T, Martin M, Doblas VG, Bacete L, Miart F, Sormani R, Hématy K, Renou J, Landrein B, et al. (2018) Receptor kinase THESEUS1 is a rapid alkalinization factor 34 receptor in Arabidopsis. Curr Biol 28: 2452-2458

Hamann T, Bennett M, Mansfield J, Somerville C (2009) Identification of cell-wall stress as a hexose-dependent and osmosensitive of plant responses. Plant J 57: 1015-1026

Hématy K, Sado P-E, van Tuinen A, Rochange S, Desnos T, Balzergue S, Pelletler S, Renoy JP, Höfte H (2007) A receptorlike kinase mediates the response of Arabidopsis cells to the inhibition of cellulose synthesis. Curr Biol 17: 922-931

Howell SH (2013) Endoplasmic reticulum stress responses in plants. Annu Rev Plant Biol 64: 477-499

Iwata Y, Koizumi N (2012) Plant transducers of the endoplasmic reticulum unfolded protein response. Trends Plant Sci 17: $720-727$

Krysan DJ (2009) The cell wall and endoplasmic reticulum stress response are coordinately regulated in Saccharomyces cerevisiae. Commun Integr Biol 2: 233-235

Liu JX, Howell SH (2016) Managing the protein folding demands in the endoplasmic reticulum of plants. New Phytol 211: 418-428

Liebminger E, Grass J, Altmann F, Mach L, Strasser R (2013) Characterizing the link between glycosylation state and enzymatic activity of the endo- $\beta 1,4$-glucanase KORRIGAN1 from Arabidopsis thaliana. J Biol Chem 288: 22270-22280

Lindner H, Müller LM, Boisson-Dernier A, Grossniklaus U (2012) CrRLK1L receptor-like kinases: Not just another brick the wall. Curr Opin Plant Biol 15: 659-669

Meijering E, Jacob M, Sarria JC, Steiner P, Hirling H, Unser M (2004) Design and validation of a tool for neurite tracing and analysis in fluorescence microscopy images. Cytometry $A$ 58: $167-176$

Merz D, Richter J, Gonneau M, Sanchez-Rodriguez C, Eder T, Sormani R, Martin M, Hématy K, Hofte H, Hauser MT (2017) T-DNA alleles of the receptor kinase THESEUS1 with opposing effects on cell wall integrity signaling. J Exp Bot 68: 4583-4593

Moreno AA, Mukhtar MS, Blanco F, Boatwright JL, Moreno I, Jordan MR, Chen Y, Brandizzi F, Dong X, Orellana A, et al. (2012) IRE1/bZIP60-mediated unfolded protein response plays distinct roles in plant immunity and abiotic stress response. PLoS One 7: e31944

Moreno AA, Orellana A (2011) The physiological role of the unfolded protein response in plants. Biol Res 44: 75-80

Murashige T, Skoog F (1962) A revised medium for rapid growth and bioassays with tobacco tissue cultures. Physiol Plant 15: 473-497

Nagashima Y, Iwata Y, Ashida M, Mishiba K, Koizumi N (2014) Exogenous salicylic acid activates two signaling arms of the unfolded protein response in Arabidopsis. Plant Cell Physiol 55: 1772-1778

Nagashima Y, von Schaewen V, Koiwa H (2018) Function of Nglycosylation in plants. Plant Sci 274: 70-79

Nakamura M, Kamehama T, Sato Y (2020) Image analysis of stress- 
induced lignin deposition in Arabidopsis thaliana using the macro program LigninJ for ImageJ software. Plant Biotechnol 37: 105-109

Nakano J, Meshitsuka G (1992) The detection of lignin. In: Dence C, Lin S (eds) Methods in Lignin Chemistry. Springer-Verlag, Berlin, pp 23-32

Nissen KS, Willats WG, Malinovsky FG (2016) Understanding CrRLK1L function: Cell walls and growth control. Trends Plant Sci 21: 516-527

Nozaki M, Sugiyama M, Duan J, Uematsu H, Genda T, Sato Y (2012) A missense mutation in the glucosamine-6-phosphate $N$ acetyltransferase-encoding gene causes temperature-dependent growth defects and ectopic lignin deposition in Arabidopsis. Plant Cell 24: 3366-3379

Ozgur R, Uzilday B, Sekmen AH, Turkan I (2015) The effects of induced production of reactive oxygen species in organelles on endoplasmic reticulum stress and on the unfolded protein response in arabidopsis. Ann Bot 116: 541-553

Rasband WS (1997-2021) ImageJ. U. S. National Institutes of Health, Bethesda, Maryland. http://rsb.info.nih.gov/ij/ (accessed on Oct. 5, 2021)

R Core Team (2021) R: A language and environment for statistical computing. R Foundation for Statistical Computing, Vienna, Austria. https://www.R-project.org/ (accessed on Oct. 5, 2021)

Ruberti C, Lai Y, Brandizzi F (2018) Recovery from temporary endoplasmic reticulum stress in plants relies on the tissuespecific and largely independent roles of bZIP28 and bZIP60, as well as an antagonizing function of BAX-Inhibitor 1 upon the pro-adaptive signaling mediated by bZIP28. Plant J 93: 155-165

Scrimale T, DiDone L, de Mesy Bentley KL, Krysan DJ (2009) The unfolded protein response is induced by the cell wall integrity mitogen-activated protein kinase pathway and required for cell wall integrity in Saccharomyces cerevisiae. Mol Biol Cell 20: 164-175

Strasser R (2014) Biological significance of complex $N$-glycans in plants and their impact on plant physiology. Front Plant Sci 5: 363

Strasser R (2018) Protein quality control in the endoplasmic reticulum of plants. Annu Rev Plant Biol 69: 147-172

Torres MA, Dangl JL (2005) Functions of the respiratory burst oxidase in biotic interactions, abiotic stress and development. Curr Opin Plant Biol 8: 397-403

van der Does D, Boutrot F, Engelsdorf T, Rhodes J, McKenna JF, Vernhettes S, Koevoets I, Tintor N, Veerabagu M, Miedes E, et al. (2017) The Arabidopsis leucine-rich repeat receptor kinase MIK2/LRR-KISS connects cell wall integrity sensing, root growth and response to abiotic and biotic stresses. PLoS Genet 13: e1006832

Yin Z, Tang W, Wang J, Liu X, Yang L, Gao C, Zhang J, Zhang H, Zheng X, Wang P, et al. (2016) Phosphodiesterase MoPdeH targets MoMck1 of the conserved mitogen-activated protein (MAP) kinase signalling pathway to regulate cell wall integrity in rice blast fungus Magnaporthe oryzae. Mol Plant Pathol 17: 654-668

Zhang M, Henquet M, Chen Z, Zhang Z, Zhang Y, Ren X, van der Krol S, Gonneau M, Bosch D, Gong Z (2009) LEW3, encoding a putative $\alpha$-1,2-mannosyltransferase (ALG11) in $N$-linked glycoprotein, plays vital roles in cell-wall biosynthesis and the abiotic stress response in Arabidopsis thaliana. Plant $J$ 60: 983-999 